\title{
Reverses and variations of the Young inequality
}

\author{
Haisong Cao*, Junliang Wu \\ College of Mathematics and Statistics, Chongqing University, Chongqing, 401331, China
}

*Corresponding author, e-mail: hscao123@163.com

Received 17 Jun 2015

Accepted 29 Dec 2015

ABSTRACT: We extend the range of the weighted operator means for $v \notin[0,1]$ and obtain some corresponding operator inequalities. We also present several reversed Young-type inequalities.

KEYWORDS: weighted operator, positive operator, binary operation, Hilbert-Schmidt norm, Young-type inequality

MSC2010: 47A30 15A60

\section{INTRODUCTION}

Let $B(H)$ be the $C^{*}$-algebra of all bounded linear operators on a Hilbert space $H$ equipped with the operator norm, $S(H)$ the set of all bounded selfadjoint operators, and $\mathbb{P}=\mathbb{P}(\mathrm{H})$ the open convex cone of all positive invertible operators. For $X, Y \in$ $S(H)$, we write $X \leqslant Y$ if $Y-X$ is positive, and $X<Y$ if $Y-X$ is positive invertible.

The unitarily invariant norm $\|\cdot\|$ is defined on the matrix algebra $\mathbb{M}_{n}$ of all $n \times n$ matrices with entries in the complex field $\mathbb{C}$. For $A=\left(a_{i j}\right) \in \mathbb{M}_{n}$, the Hilbert-Schmidt norm of $A$ is defined by $\|A\|_{2}=$ $\left(\sum_{j=1}^{n} s_{j}^{2}(A)\right)^{1 / 2}$, where $s_{1}(A), s_{2}(A), \ldots, s_{n}(A)$ are the singular values of $A$, i.e., the eigenvalues of the positive matrix $|A|=\left(A^{*} A\right)^{1 / 2}$ where $\left.A^{*}=(\bar{A})^{\mathrm{T}}\right)$, arranged in decreasing order and repeated according to multiplicity. It is known that the Hilbert-Schmidt norm is unitarily invariant.

Let $a, b>0$ be two positive real numbers and $v \in[0,1]$. The $v$-weighted arithmetic and geometric means of $a$ and $b$, denoted by $A_{v}(a, b)$ and $G_{v}(a, b)$, respectively, are defined as

$$
A_{v}(a, b)=(1-v) a+v b, \quad G_{v}(a, b)=a^{1-v} b^{v} .
$$

Note that $A_{v}(a, b) \geqslant G_{v}(a, b)$ for all $v \in[0,1]$. This is the well-known Young inequality. In particular, if $v=\frac{1}{2}$ then $A_{1 / 2}(a, b)=\frac{1}{2}(a+b)$ and $G_{1 / 2}(a, b)=$ $\sqrt{a b}$ are the arithmetic and geometric means, respectively. The Heinz mean of $a$ and $b$ is defined as

$$
H_{v}(a, b)=\frac{a^{v} b^{1-v}+a^{1-v} b^{v}}{2}
$$

for $v \in[0,1]$. For $v=0,1$, this is equal to arithmetic mean and for $v=\frac{1}{2}$ it is the geometric mean.
Let $A, B \in B(H)$ be two positive operators and $v \in[0,1]$. The $v$-weighted arithmetic mean of $A$ and $B$, denoted by $A \nabla_{v} B$, is defined as

$$
A \nabla_{v} B=(1-v) A+v B .
$$

If $A$ is invertible, the $v$-weighted geometric mean of $A$ and $B$, denoted by $A \sharp_{v} B$, is defined as

$$
A \sharp_{v} B=A^{1 / 2}\left(A^{-1 / 2} B A^{-1 / 2}\right)^{v} A^{1 / 2} .
$$

For more details, see Ref. 1 . When $v=\frac{1}{2}$, we write $A \nabla B$ and $A \sharp B$ for brevity, respectively.

The operator version of the Heinz mean, denoted by $H_{v}(A, B)$, is defined as

$$
H_{v}(A, B)=\frac{A \sharp_{v} B+A \sharp_{1-v} B}{2}, \quad 0 \leqslant v \leqslant 1 .
$$

It is well known that if $A$ and $B$ are positive invertible operators, then

$$
A \nabla_{v} B \geqslant A \sharp_{v} B, \quad 0 \leqslant v \leqslant 1 .
$$

The Specht ratio ${ }^{2,3}$ is defined by

$$
S(t)=\frac{t^{1 /(t-1)}}{\operatorname{elog} t^{1 /(t-1)}} \text { for } t>0, t \neq 1,
$$

and

$$
S(1)=\lim _{t \rightarrow 1} S(t)=1 \text {. }
$$

Furuichi ${ }^{4}$ gave the following refined version:

$$
A \nabla_{v} B \geqslant S\left(h^{r}\right) A \sharp_{v} B \geqslant A_{\sharp_{v}} B,
$$

where $r=\min \{v, 1-v\}$. Zuo et $\mathrm{al}^{5}$ gave another one:

$$
K(h, 2)^{r} A \sharp_{v} B \leqslant A \nabla_{v} B,
$$


where $K(t, 2)=(t+1)^{2} / 4 t$ for $t>0$ is the Kantorovich constant. In Ref. 6, Furuichi gave another refined version:

$$
A \nabla_{\nu} B \geqslant A \sharp_{v} B+2 r(A \nabla B-A \sharp B) \geqslant A \sharp_{v} B .
$$

Recently there have been a number of other studies on similar topics and various improvement versions ${ }^{7-11}$.

The Heinz norm inequality, which is one of the essential inequalities in operator theory, states that for any positive operators $A, B \in M_{n}$, any operator $X \in M_{n}$ and $v \in[0,1]$, the following double inequality holds:

$$
\begin{aligned}
2\left\|A^{1 / 2} X B^{1 / 2}\right\| \leqslant \| A^{v} X B^{1-v}+ & A^{1-v} X B^{v} \| \\
& \leqslant\|A X+X B\| .
\end{aligned}
$$

Kittaneh and Manasrah ${ }^{12}$ showed a refinement of the right-hand side of inequality (1) for the Hilbert-Schmidt norm as follows:

$$
\begin{array}{r}
\left\|A^{v} X B^{1-v}+A^{1-v} X B^{v}\right\|_{2}^{2}+2 r_{0}\|A X-X B\|_{2}^{2} \\
\leqslant\|A X+X B\|_{2}^{2},
\end{array}
$$

in which $A, B, X \in M_{n}$ such that $A, B$ are positive semidefinite, $v \in[0,1]$ and $r_{0}=\min \{v, 1-v\}$. Kaur et $\mathrm{al}^{13}$, using the convexity of the function $f(v)=$ $\left\|A^{v} X B^{1-v}+A^{1-v} X B^{v}\right\| \mid$ with $v \in[0,1]$, presented more refinements of the Heinz inequality.

It was shown in Ref. 14 that a reverse of inequality (2) is

$$
\begin{aligned}
\|A X+X B\|_{2}^{2} \leqslant \| A^{v} X B^{1-v} & +A^{1-v} X B^{v} \|_{2}^{2} \\
& +2 r_{0}\|A X-X B\|_{2}^{2},
\end{aligned}
$$

where $A, B, X \in M_{n}$ such that $A, B$ are positive semidefinite, $v \in[0,1]$, and $r_{0}=\max \{v, 1-v\}$.

In this paper, we extend the range of the weighted operator means for $v \notin[0,1]$ and obtain some corresponding operator inequalities. We also present a reverse of (2) and some other operator inequalities.

\section{SOME OPERATOR INEQUALITIES FOR $v \notin[0,1]$}

For $A, B \in \mathbb{P}$ and $v \in[0,1]$, the $v$-weighted geometric operator mean is defined as

$$
A \sharp_{v} B=A^{1 / 2}\left(A^{-1 / 2} B A^{-1 / 2}\right)^{v} A^{1 / 2} .
$$

For convenience, we use the notation $\bigsqcup_{v}$ and $H_{v}^{\natural}$ for the binary operation

$$
\begin{gathered}
A \bigsqcup_{v} B=A^{1 / 2}\left(A^{-1 / 2} B A^{-1 / 2}\right)^{v} A^{1 / 2}, \\
H_{v}^{\natural}(A, B)=\frac{A \natural_{v} B+A \natural_{1-v} B}{2},
\end{gathered}
$$

for $v \notin[0,1]$. We use the notation $\diamond_{v}$ and $H_{v}$ for the binary operation

$$
\begin{gathered}
A \diamond_{\nu} B=A^{1 / 2}\left(A^{-1 / 2} B A^{-1 / 2}\right)^{v} A^{1 / 2}, \\
H_{v}^{\diamond}(A, B)=\frac{A \bigsqcup_{v} B+A \bigsqcup_{1-v} B}{2},
\end{gathered}
$$

for $v \notin\left[\frac{1}{2}, 1\right]$, whose formulae are the same as $\sharp_{v}$ and $H_{v}(A, B)$. Note that $A \sharp_{v} B$ for $v \in[0,1]$ is monotonic, but $A \natural_{v} B$ and $A \diamond_{v} B$ are not.

In this section, we extend the range of the definition of the weighted operator. We also present some operator inequalities for $v \notin[0,1]$ and $v \notin$ $\left[\frac{1}{2}, 1\right]$. To obtain the results, we need the following lemmas.

Lemma 1 (Ref. 15) Let $X \in B(H)$ be self-adjoint and let $f$ and $g$ be continuous real functions such that $f(t) \geqslant g(t)$ for all $t \in \mathrm{Sp}(X)$ (the spectrum of $X$ ). Then $f(X) \geqslant g(X)$.

Lemma 2 (Ref. 16) Let $a, b>0$ and $v \notin[0,1]$. Then,

(i)

$$
v a+(1-v) b+(v-1)(\sqrt{a}-\sqrt{b})^{2} \leqslant a^{v} b^{1-v},
$$

(ii)

$$
(a+b)+2(v-1)(\sqrt{a}-\sqrt{b})^{2} \leqslant a^{v} b^{1-v}+b^{v} a^{1-v},
$$

$$
(a+b)^{2}+2(v-1)(a-b)^{2} \leqslant\left(a^{v} b^{1-v}+b^{v} a^{1-v}\right)^{2} .
$$

Proof: Let $a, b>0$ and $v \notin[0,1]$.

(i) Assume that $f(t)=t^{1-v}-v+(v-1) t$ with $t \in$ $(0, \infty)$. It is easy to see that $f(t)$ has a minimum at $t=1$ in the interval $(0, \infty)$. Hence $f(t) \geqslant f(1)=0$ for all $t>0$. Assume that $a, b>0$. Letting $t=b / a$, we get

$$
v a+(1-v) b \leqslant a^{v} b^{1-v} .
$$

So we have

$$
\begin{aligned}
v a & +(1-v) b+(v-1)(\sqrt{a}-\sqrt{b})^{2} \\
& =(2-2 v) \sqrt{a b}+(2 v-1) a \\
& \leqslant(\sqrt{a b})^{2-2 v} a^{2 v-1}=a^{v} b^{1-v} .
\end{aligned}
$$

(ii) It can be proved in a similar fashion to (i).

(iii) It follows from (ii) by replacing $a$ by $a^{2}$ and $b$ by $b^{2}$. 
Theorem 1 Let $A, B \in \mathbb{P}$ and $v \notin[0,1]$. Then:

$$
v A+(1-v) B+2(v-1)(A \nabla B-A \sharp B) \leqslant A \natural_{1-v} B .
$$

Proof: By Lemma 2(i), we have

$$
v+(1-v) b+(v-1)(1-\sqrt{b})^{2} \leqslant b^{1-v},
$$

for any $b>0$. If $X=A^{-1 / 2} B A^{-1 / 2}$ and thus $\operatorname{Sp}(X) \subseteq$ $(0,+\infty)$, then we have

$$
v+(1-v) t+(v-1)(1-\sqrt{t})^{2} \leqslant t^{1-v},
$$

for any $t \in \operatorname{Sp}(X)$. This is the same as

$$
v I+(1-v) X+(v-1)\left(I-X^{1 / 2}\right)^{2} \leqslant X^{1-v} .
$$

Multiplying both sides of (4) by $A^{1 / 2}$, we get

$$
\begin{gathered}
v A+(1-v) B+(v-1)\left(A+B-2 A^{1 / 2} X^{1 / 2} A^{1 / 2}\right) \\
\leqslant A^{1 / 2} X^{1-v} A^{1 / 2}
\end{gathered}
$$

If $v \notin[0,1]$, then

$$
v A+(1-v) B+2(v-1)(A \nabla B-A \sharp B) \leqslant A \natural_{1-v} B .
$$

Remark 1 In Ref. 12, the authors showed that if $v \in$ $\left(0, \frac{1}{2}\right)$, then

$$
v A+(1-v) B+2(v-1)(A \nabla B-A \sharp B) \leqslant A \sharp_{1-v} B .
$$

It is the same version of the formula (5). Hence for all $v \notin\left[\frac{1}{2}, 1\right]$,

$$
v A+(1-v) B+2(v-1)(A \nabla B-A \sharp B) \leqslant A \diamond_{1-v} B
$$

holds.

Remark 2 If $A, B \in \mathbb{P}$ and $B \geqslant A, v \in(1,2)$, then by the monotonicity of $\sharp_{v}$ and $0<v-1<1, B^{-1} \leqslant A^{-1}$,

$$
\begin{aligned}
v A & +(1-v) B+2(v-1)(A \nabla B-A \sharp B) \leqslant A \natural_{1-v} B \\
& =A^{1 / 2}\left(A^{-1 / 2} B A^{-1 / 2}\right)^{1-v} A^{1 / 2} \\
& =A^{1 / 2}\left(A^{1 / 2} B^{-1} A^{1 / 2}\right)^{v-1} A^{1 / 2} \\
& \leqslant A^{1 / 2}\left(A^{1 / 2} A^{-1} A^{1 / 2}\right)^{v-1} A^{1 / 2}=A .
\end{aligned}
$$

This is the same as

$$
0 \leqslant A \nabla B-A \sharp B \leqslant \frac{B-A}{2} .
$$

By Lemma 2 (ii), (iii) and using the same processing technique as in Theorem 1, we can get the following theorems and the corresponding remarks.
Theorem 2 Let $A, B \in \mathbb{P}$ and $v \notin[0,1]$. Then

$$
A \nabla B+2(v-1)(A \nabla B-A \sharp B) \leqslant H_{v}^{\natural}(A, B) .
$$

Remark 3 In Ref. 14, the authors showed that if $v \in$ $\left(0, \frac{1}{2}\right)$, then

$$
A \nabla B+2(v-1)(A \nabla B-A \sharp B) \leqslant H_{v}(A, B) .
$$

Hence for all $v \notin\left[\frac{1}{2}, 1\right]$,

$$
A \nabla B+2(v-1)(A \nabla B-A \sharp B) \leqslant H_{v}^{\diamond}(A, B)
$$

holds.

Remark 4 If $A, B \in \mathbb{P}$ and $B \geqslant A, v \in(1,2)$, then

$$
B+4(v-1)(A \nabla B-A \sharp B) \leqslant A \natural_{v} B .
$$

Theorem 3 Let $A, B \in \mathbb{P}$ and $v \notin[0,1]$. Then

$$
(2 v-1)\left(A+A \natural_{2} B\right)-4(v-1) B \leqslant A \natural_{2-2 v} B+A \natural_{2 v} B .
$$

Remark 5 If $A, B \in \mathbb{P}$ and $B \geqslant A, v \in(1,2)$, then

$$
2(v-1)(A-2 B)+(2 v-1) A \natural_{2} B \leqslant A \natural_{2 v} B .
$$

\section{A REVERSE OF THE HEINZ INEQUALITY FOR MATRICES}

In this section, we present a reverse of the Heinz inequality for matrices. To obtain the result, we need the following lemma.

Lemma 3 (Ref. 17) Let $a, b>0$. If $0 \leqslant v \leqslant \frac{1}{2}$, then

$$
\begin{aligned}
v^{2} a+(1-v)^{2} b \leqslant(1-v)^{2} & (\sqrt{a}-\sqrt{b})^{2} \\
+ & a^{v}\left[(1-v)^{2} b\right]^{1-v} .
\end{aligned}
$$

If $\frac{1}{2} \leqslant v \leqslant 1$, then

$$
v^{2} a+(1-v)^{2} b \leqslant v^{2}(\sqrt{a}-\sqrt{b})^{2}+\left(v^{2} a\right)^{v} b^{1-v} .
$$

Based on Lemma 3, the following corollaries can be easily obtained.

Corollary 1 Let $a, b>0$. If $0 \leqslant v \leqslant \frac{1}{2}$, then

$$
\begin{aligned}
2 v(a+b) \leqslant & 2(1-v)(\sqrt{a}-\sqrt{b})^{2} \\
& +(1-v)^{1-2 v}\left[a^{v} b^{1-v}+b^{v} a^{1-v}\right] .
\end{aligned}
$$

If $\frac{1}{2} \leqslant v \leqslant 1$, then

$$
\begin{aligned}
2(1-v)(a+b) \leqslant & 2 v(\sqrt{a}-\sqrt{b})^{2} \\
& +v^{2 v-1}\left[a^{v} b^{1-v}+b^{v} a^{1-v}\right] .
\end{aligned}
$$


Corollary 2 Let $a, b>0$. If $0 \leqslant v \leqslant \frac{1}{2}$, then

$$
\begin{aligned}
& \begin{aligned}
2 v(a+b)^{2} & \leqslant 2(1-v)(a-b)^{2} \\
& +(1-v)^{1-2 v}\left(a^{v} b^{1-v}+b^{v} a^{1-v}\right)^{2} . \\
\text { If } \frac{1}{2} \leqslant v \leqslant 1, \text { then } & \\
2(1-v)(a+b)^{2} & \leqslant 2 v(a-b)^{2} \\
& +v^{2 v-1}\left(a^{v} b^{1-v}+b^{v} a^{1-v}\right)^{2} .
\end{aligned}
\end{aligned}
$$

Theorem 4 Let $A, B, X \in \mathbb{M}_{n}$ with $A, B$ are positive, and $v \in[0,1]$. Then

$$
\begin{aligned}
2 v \| A X+ & X B\left\|_{2}^{2} \leqslant 2(1-v)\right\| A X-X B \|_{2}^{2} \\
& +(1-v)^{1-2 v}\left\|A^{v} X B^{1-v}+A^{1-v} X B^{v}\right\|_{2}^{2}
\end{aligned}
$$

for $0 \leqslant v \leqslant \frac{1}{2}$, and

$$
\begin{aligned}
2(1-v) \| A X+ & X B\left\|_{2}^{2} \leqslant 2 v\right\| A X-X B \|_{2}^{2} \\
& +v^{2 v-1}\left\|A^{v} X B^{1-v}+A^{1-v} X B^{v}\right\|_{2}^{2}
\end{aligned}
$$

for $\frac{1}{2} \leqslant v \leqslant 1$.

Proof: By spectral decomposition, there are unitary matrices $U, V \in \mathbb{M}_{n}$ such that $A=U \Lambda_{1} U^{*}$ and $B=$ $V \Lambda_{2} V^{*}$, where

$$
\Lambda_{1}=\operatorname{diag}\left(\lambda_{1}, \lambda_{2}, \ldots, \lambda_{n}\right)
$$

and

$$
\Lambda_{2}=\operatorname{diag}\left(\mu_{1}, \mu_{2}, \ldots, \mu_{n}\right)
$$

where $\lambda_{i}$ and $\mu_{i}$ for $i=1,2, \ldots, n$ are the eigenvalues of $A$ and $B$, respectively. Let $Y=U^{*} X V=\left[y_{i j}\right]$, then

$$
\begin{aligned}
& A X+X B=U\left(\Lambda_{1} Y+Y \Lambda_{2}\right) V^{*} \\
& =U\left[\left(\lambda_{i}+\mu_{i}\right) y_{i j}\right] V^{*}, \\
& A X-X B=U\left(\Lambda_{1} Y-Y \Lambda_{2}\right) V^{*} \\
& =U\left[\left(\lambda_{i}-\mu_{i}\right) y_{i j}\right] V^{*}, \\
& A^{v} X B^{1-v}+A^{1-v} X B^{v} \\
& =U \Lambda_{1}^{v} U^{*} X V \Lambda_{2}^{1-v} V^{*}+U \Lambda_{1}^{1-v} U^{*} X V \Lambda_{2}^{v} V^{*} \\
& =U \Lambda_{1}^{v} Y \Lambda_{2}^{1-v} V^{*}+U \Lambda_{1}^{1-v} Y \Lambda_{2}^{v} V^{*} \\
& =U\left[\Lambda_{1}^{v} Y \Lambda_{2}^{1-v}+\Lambda_{1}^{1-v} Y \Lambda_{2}^{v}\right] V^{*} \\
& =U\left[\left(\lambda_{i}^{v} \mu_{i}^{1-v}+\lambda_{i}^{1-v} \mu_{i}^{v}\right) y_{i j}\right] V^{*} .
\end{aligned}
$$

If $0 \leqslant v \leqslant \frac{1}{2}$, then by (10) and the unitary invariance of the Hilbert-Schmidt norm, we have

$$
\begin{aligned}
2 v & \|A X+X B\|_{2}^{2}=2 v \sum_{i, j=1}^{n}\left(\lambda_{i}+\mu_{i}\right)^{2}\left|y_{i j}\right|^{2} \\
\leqslant & 2(1-v) \sum_{i, j=1}^{n}\left(\lambda_{i}-\mu_{i}\right)^{2}\left|y_{i j}\right|^{2} \\
& +(1-v)^{1-2 v} \sum_{i, j=1}^{n}\left(\lambda_{i}^{v} \mu_{i}^{1-v}+\lambda_{i}^{1-v} \mu_{i}^{v}\right)^{2}\left|y_{i j}\right|^{2} \\
= & 2(1-v)\|A X-X B\|_{2}^{2} \\
& +(1-v)^{1-2 v}\left\|A^{v} X B^{1-v}+A^{1-v} X B^{v}\right\|_{2}^{2} .
\end{aligned}
$$

If $\frac{1}{2} \leqslant v \leqslant 1$, then by (11) and using the same technique in the first part we get the other result.

\section{SOME REVERSES OF THE YOUNG-TYPE INEQUALITY FOR OPERATORS}

In this section, we obtain some reverses of the Young-type inequality for two positive invertible operators.

Theorem 5 Let $A, B \in \mathbb{P}$ and $v \in[0,1]$. Then

$$
\begin{aligned}
& v^{2} A+(1-v)^{2} B \leqslant 2(v-1)^{2}(A \nabla B-A \sharp B) \\
&+(1-v)^{2(1-v)} A \sharp_{1-v} B,
\end{aligned}
$$

for $0 \leqslant v \leqslant \frac{1}{2}$, and

$$
v^{2} A+(1-v)^{2} B \leqslant 2 v^{2}(A \nabla B-A \sharp B)+v^{2 v} A \sharp_{1-\nu} B,
$$

for $\frac{1}{2} \leqslant v \leqslant 1$.

Proof: For $0 \leqslant v \leqslant \frac{1}{2}$, by (6) we have

$v^{2} a+(1-v)^{2} b \leqslant(1-v)^{2}(\sqrt{a}-\sqrt{b})^{2}+a^{v}\left[(1-v)^{2} b\right]^{1-v}$, for any $b>0$. If $X=A^{-1 / 2} B A^{-1 / 2}$ and thus $\operatorname{Sp}(X) \subseteq$ $(0,+\infty)$, then we have

$v^{2}+(1-v)^{2} b \leqslant(1-v)^{2}(1-\sqrt{b})^{2}+\left[(1-v)^{2} b\right]^{1-v}$,

for any $t \in \operatorname{Sp}(X)$. This is the same as

$v^{2} I+(1-v)^{2} X \leqslant(1-v)^{2}\left(I-X^{1 / 2}\right)^{2}+\left[(1-v)^{2} X\right]^{1-v}$.

Multiplying both sides of (12) by $A^{1 / 2}$, we get

$$
\begin{aligned}
v^{2} A+(1-v)^{2} B \leqslant 2(v-1)^{2}(A \nabla B-A \sharp B) \\
+(1-v)^{2(1-v)} A \sharp_{1-v} B .
\end{aligned}
$$


Theorem 6 Let $A, B \in \mathbb{P}$ and $v \in[0,1]$. Then

$2 v A \nabla B \leqslant 2(1-v)(A \nabla B-A \sharp B)+(1-v)^{1-2 v} H_{v}(A, B)$,

for $0 \leqslant v \leqslant \frac{1}{2}$, and

$2(1-v) A \nabla B \leqslant 2 v(A \nabla B-A \sharp B)+v^{2 v-1} H_{v}(A, B)$,

for $\frac{1}{2} \leqslant v \leqslant 1$.

Proof: By Corollary 2 and the same processing technique as in Theorem 5, we can easily obtain the result.

\section{REFERENCES}

1. Kubo F, Ando T (1980) Means of positive operators. Math Ann 264, 205-24.

2. Fujii JI, Izumino S, Seo Y (1998) Determinant for positive operators and Specht's Theorem. Sci Math Japon 1, 307-10.

3. Specht W (1960) Zur Theorie der elementaren Mittel. Math Z 74, 91-8.

4. Furuichi S (2012) Refined Young inequalities with Specht's ratio. J Egypt Math Soc 20, 46-9.

5. Zuo HL, Shi GH, Fujii M (2011) Refined Young inequality with Kantorovich constant. $J$ Math Inequal 5, 551-6.

6. Furuichi S (2011) On refined Young inequalities and reverse inequalities. $J$ Math Inequal 5, 21-31.

7. Kittaneh F, Krnić M, Lovričević N, Pečarić J (2012) Improved arithmetic-geometric and Heinz means inequalities for Hilbert space operators. Publ Math Debrecen 80, 465-78.

8. Krnić M, Lovričević N, Pečarić J (2012) Jensen's operator and applications to mean inequalities for operators in Hilbert space. Bull Malays Math Sci Soc 35, 1-14.

9. Zhao JG, Wu JL, Cao HS, Liao WS (2014) Operator inequalities involving the arithmetic, geometric, Heinz and Heron means. J Math Inequal 8, 747-56.

10. Hirzallah O, Kittaneh F, Krnić M, Lovričević N, Pečarić $\mathrm{J}$ (2012) Eigenvalue inequalities for differences of means of Hilbert space operators. Lin Algebra Appl 436, 1516-27.

11. Hu XK, Xue JM (2015) A note on reverses of Young type inequalities. J Inequal Appl 98, 1-6.

12. Kittaneh F, Manasrah Y (2010) Improved Young and Heinz inequalities for matrices. $J$ Math Anal Appl 361, 262-9.

13. Kaur R, Manasrah MS, Singh M, Conde C (2014) Further refinements of Heinz inequality. Lin Algebra Appl 447, 26-37.

14. Kittaneh F, Manasrah Y (2011) Reverse Young and Heinz inequalities for matrices. Lin Multilin Algebra 59, 1031-7.

15. Wu JL, Zhao JG (2014) Operator inequalities and reverse inequalities related to the Kittaneh-Manasrah inequalities. Lin Multilin Algebra 62, 884-94.
16. Mojtaba B, Mohammad SM (2015) Reverses and variations of Heinz inequality. Lin Multilin Algebra 63, 1972-80.

17. Burqan A, Khandaqji M (2015) Reverses of Young type inequalities. J Math Inequal 9, 113-20. 